\title{
Rapid changes in visual-spatial attention distort object shape
}

\author{
Francesca C. Fortenbaugh • William Prinzmetal • \\ Lynn C. Robertson
}

Published online: 11 February 2011

(C) The Author(s) 2011. This article is published with open access at Springerlink.com

\begin{abstract}
Shifts of attention due to rapid cue onsets have been shown to distort the perceived location of objects, but are there also systematic distortions in the perceived shapes of the objects themselves from such shifts? The present study demonstrates that there are. In three experiments, oval contours were presented that varied in width and height. Two brief, bright white dots were presented as cues and were positioned horizontally or vertically either inside or outside the oval contour. Observers had to judge whether the oval was taller than wide. The results show that the perceived shape of an oval was changed by visual cues such that the oval contours were repelled by the cues (Exp. 1). This effect only occurred when the cues preceded the ovals, providing sufficient time between the presentations to attract involuntary attention (Exp. 2). Moreover, an explanation based on figural aftereffects was ruled out (Exp. 3).
\end{abstract}

Keywords Attentional repulsion effect - Spatial attention . Involuntary attention Shape perception

Research has shown that changes in the focus or distribution of spatial attention alter not only how quickly observers are able to detect target items in a display (Jonides, 1980; Posner, Snyder, \& Davidson, 1980) but also the perceived location of subsequently presented targets

F. C. Fortenbaugh · L. C. Robertson

Veterans Administration,

Martinez, CA, USA

F. C. Fortenbaugh $\cdot$ W. Prinzmetal $\cdot$ L. C. Robertson $(\square)$

Department of Psychology, University of California,

4143 Tolman Hall \#5050,

Berkeley, CA 94720, USA

e-mail: lynnrob@berkeley.edu
(Adam, Davelaar, van der Gouw, \& Willems, 2008; Tsal \& Bareket, 1999). For instance, Suzuki and Cavanagh (1997) demonstrated that the perceived offset of two vertically oriented vernier lines (arranged above and below fixation) can be systematically displaced when a pair of circular, noninformative cues are laterally presented on opposite sides of the two vernier lines prior to their onset. The displacement was also found when a single attentional cue was used. This effect is called the attentional repulsion effect (ARE) because the perceived displacement of the top vernier line relative to the bottom one was found to be in the direction away from the cued locations.

The present study asked a different but complementary question-namely, can involuntary attention alter the perception of an object's shape? To address this question, we modified the ARE paradigm by replacing the two vernier lines with a single large, oval contour that varied in height, and asked participants to determine whether each oval was wider or taller than a perfect circle. Prior to presenting the oval, two small white dots (cues) were flashed along either the horizontal or vertical meridian, and were located either inside or outside the contour of the subsequently presented oval.

Figure 1 represents the predictions if attention alters the perceived shape of an oval contour and the cues selectively repel the part of the contour closest to the cue locations (i.e., an ARE on shape perception). When the cues are along the horizontal meridian and inside the oval, this should cause the oval to appear wider than it actually is (dashed line). When the cues are outside the oval along the horizontal meridian, the oval should appear taller than it is. Importantly, vertical cues should lead to the opposite perception (i.e., outside cues making the oval look wider and inside cues making the oval look taller). 


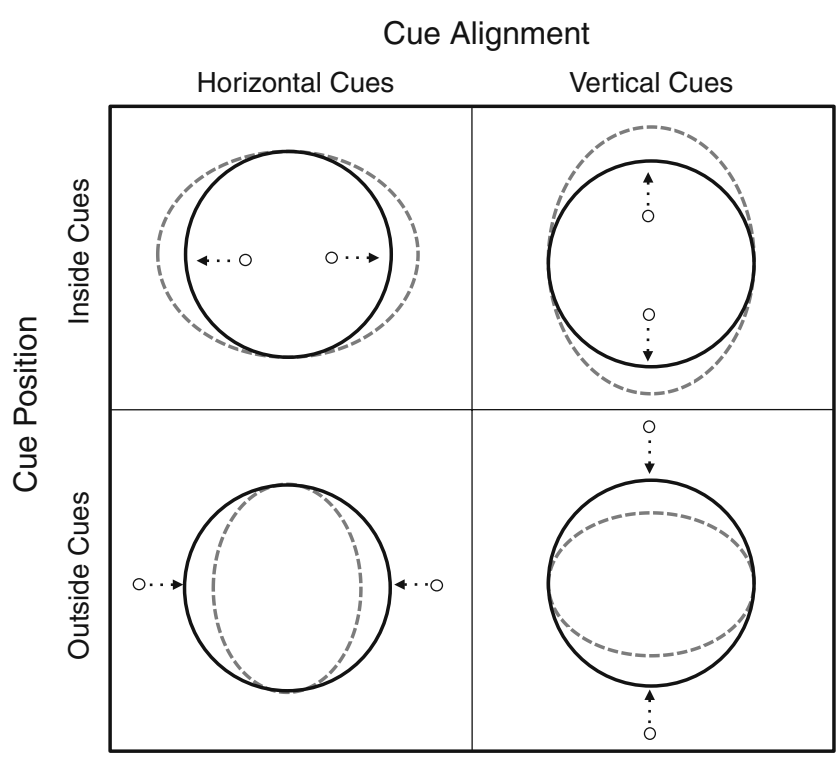

Fig. 1 Experimental predictions: Schematic representation of the predicted distortions if an attentional repulsion effect changes perceived shape. The columns represent cue alignment along the horizontal and vertical meridians, while the rows represent the cue positions inside or outside of the oval contour. The small dots represent the cues, and the large solid ovals represent the oval presented to participants. The dashed arrows represent the predicted direction of the repulsion away from the cued locations, and the dashed oval contours represent the percept. The figure is not drawn to scale

\section{Experiment 1}

\section{Method}

Participants Thirteen undergraduates (10 female; mean age $=22.7 \pm 4.03$ years) participated for course credit. All reported normal or corrected-to-normal vision and no ocular disorders. Glasses, astigmatism, or any indication of ocular disease were criteria for exclusion. One participant was an outlier, showing no sensitivity to physical changes in the height of the ovals (chance performance), and was removed from all analyses.

Materials and procedure Participants sat $25.4 \mathrm{~cm}$ from the monitor (ViewSonic G225f, refresh rate $=100 \mathrm{~Hz}$ ). Head position was stabilized with a chin-and-forehead rest. A large black piece of cardboard with a cut-out circular aperture $\left(14.67 \mathrm{~cm}\right.$, or $30^{\circ}$ radius) was centered over the screen to eliminate any influences from the lines and angles of the monitor itself. The experiment was run in a dark room. The fixation cross was centered both laterally and vertically so that the participant looked straight ahead.

The experiments were controlled with MATLAB software using the Psychophysics Toolbox (Brainard, 1997). Figure 2 shows an example trial sequence. A blue fixation cross $\left(1^{\circ}\right.$ of visual angle, $\left.10 \mathrm{~cd} / \mathrm{m}^{2}\right)$ first appeared for $500 \mathrm{~ms}$

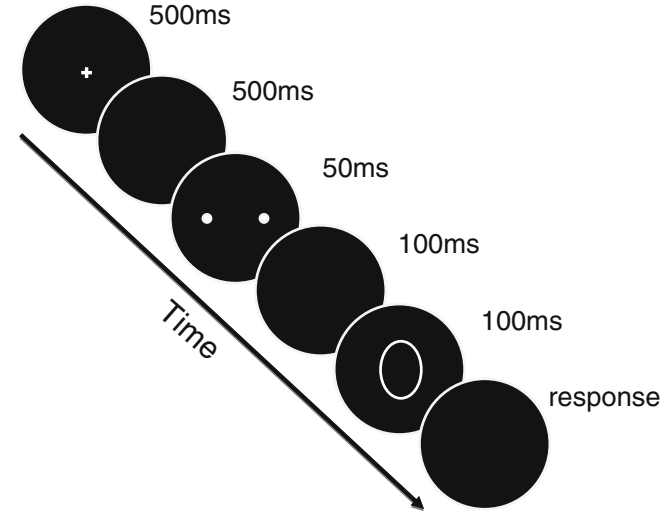

Fig. 2 Illustration of a trial in Experiment 1: An exemplary trial that shows the timing of the cue and target presentations. The display was viewed inside a circular aperture (shown as a black circle here). The cue pair and oval are not drawn to scale

on a black background $\left(0.3 \mathrm{~cd} / \mathrm{m}^{2}\right)$. After a $500-\mathrm{ms}$ blank screen, the cues, two white dots $\left(1^{\circ}\right.$ diameter, $\left.84 \mathrm{~cd} / \mathrm{m}^{2}\right)$, appeared for $50 \mathrm{~ms}$ along the horizontal or vertical meridian. The dots were both located at a distance of either $8^{\circ}$ or $14^{\mathrm{o}}$ from fixation. After a $100-\mathrm{ms}$ blank, a blue oval (line thickness $=0.08^{\circ}, 10 \mathrm{~cd} / \mathrm{m}^{2}$ ) was presented for $100 \mathrm{~ms}$. A two-alternative forced choice task was used: The participants judged whether the oval was wider or taller than a perfect circle by pressing the left or the up arrow on the keyboard.

Fifteen ovals with three different horizontal radii $\left(5^{\circ}, 11^{\circ}\right.$, or $17^{\circ}$ ) were used so that participants could not predict whether the subsequent oval contour would be inside or outside the cued locations. For each radius, 5 different ovals of varying relative heights were created; the relative heights differed from the widths by $0 \%, \pm 5 \%$, or $\pm 10 \%$. Thus, 2 of the ovals were wider than a perfect circle, 2 were taller than a perfect circle, and 1 was a perfect circle. A total of 40 stimulus combinations were created from the four cues (two cue eccentricities, $8^{\circ}$ or $14^{\circ}$, and two cue alignments, horizontal or vertical) and the 15 ovals. The ovals were paired with the cues such that the $8^{\circ}$ cues could only be followed by 1 of the 10 ovals with a $5^{\circ}$ or $11^{\circ}$ horizontal radius, and the $14^{\circ}$ cues could only be followed by 1 of the 10 ovals with an $11^{\circ}$ or $17^{\circ}$ horizontal radius. Therefore, for any given cue, participants could not predict whether the cues would fall inside or outside the following oval contour. Moreover, since all five oval heights were used, the orientation of the cues could not be used to predict whether the following oval would be wider or taller than a perfect circle. The critical ovals were the 5 with an $11^{\circ}$ radius, since these were the only ovals that were paired with all four sets of cues, two sets inside the oval contour $\left(8^{\circ}\right)$ and the other two outside $\left(14^{\circ}\right)$. All of the other ovals were controls to assure that no contingency existed between the cues and ovals. 
Participants were informed before beginning the experiment that the locations of the cues were not informative of which dimension was longer. All participants completed 10 randomly chosen practice trials before beginning the experiment. Twenty-five repeats of each cue-oval combination were included, for a total of 1,000 trials completed over four blocks.

\section{Results and discussion}

For every participant, the percentage of "taller" responses was calculated for each condition. These were analyzed in a 2 (cue alignment: horizontal/vertical) x 2 (cue position: inside/outside) x 5 (relative height: $0 \%, \pm 5 \%$, and $\pm 10 \%$ ) repeated measures ANOVA. The Greenhouse-Geisser correction was applied when appropriate.

As seen in Fig. 3a, participants increasingly responded that the oval was taller as the height of the oval increased, $F(1.84,20.28)=179.41, p<.001, \eta^{2}=.94$, as would be expected. There was no main effect of cue position, $F(1,11)=0.47, p=.51, \eta^{2}=.04$, or cue alignment, $F(1,11)=3.15, p=.10, \eta^{2}=.23$.

Most importantly, there was a significant Cue Position $\mathrm{x}$ Cue Alignment interaction, $F(1,11)=8.46, p=.01, \eta^{2}=.44$, and a significant three-way interaction, $F(1.35,14.88)=$ $5.50, p=.03, \eta^{2}=.33$, reflecting the crossover with cue position that can be seen in Fig. 3a across the two cue alignment conditions. The crossover shows the expected change in the perceived height of the oval as the cues are moved inside or outside the oval contour. However, the three-way interaction also shows that the effect of cue placement is not apparent when the ovals are most elongated along either dimension. Instead, the interaction in the means is most obvious when the oval is a true circle (relative height $=0 \%$ ). Repulsion effects in the original study by Suzuki and Cavanagh (1997) were found to be small, on the order of 10 arcmin of visual angle, or 1/6 of a degree. In the present study, each $5 \%$ change in the height of the $11^{\circ}$ horizontal radius ovals corresponds to $0.55^{\circ}$, roughly three times the average repulsion effect found in that previous study. Since the physical changes in the oval's height are larger than the reported magnitude of the repulsion effect, the present effects are consistent with Suzuki and Cavanagh's (1997) findings, in that effects are only seen when the height and the width are similar.

To further explore the Cue Position x Cue Alignment interaction, the individual participants' psychometric functions were fit with cumulative Gaussian distribution functions (GraphPad Prism; GraphPad Software, Inc.). This allowed an estimate of the point of subjective equality (PSE), or the relative height at which the oval appeared to be a perfect circle. These data were well fit by cumulative Gaussian distributions (average $R^{2}=.98$, range $=.80-1.0$ ). Figure $3 \mathrm{~b}$ shows the PSEs for the two cue positions when the cues were aligned along the horizontal and vertical meridians. A repeated measures ANOVA on the estimated PSE shows a significant Cue Alignment $x$ Cue Position interaction, $F(1,11)=5.38, p=.04, \eta^{2}=.33$. For the horizontally aligned cues, ovals needed to be taller than a perfect circle to be seen as a perfect circle. However, a larger shift was observed when the cues were placed inside the oval contour, consistent with the cues inside the circle repelling the contour out and making the oval look wider relative to when the cues were placed outside the contour. Consistently, the opposite effect was found for vertically a

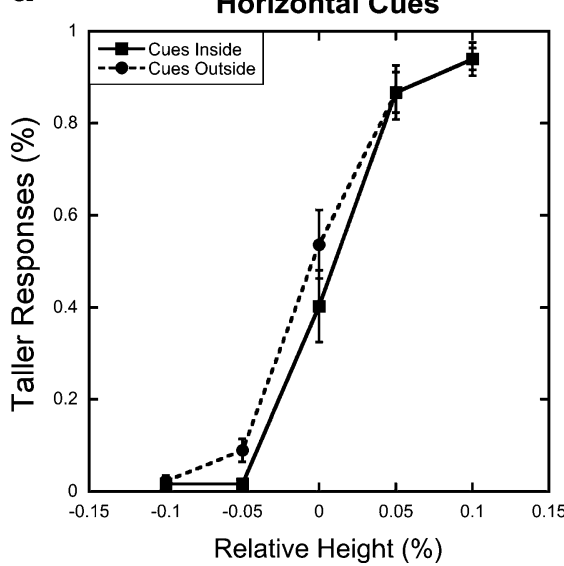

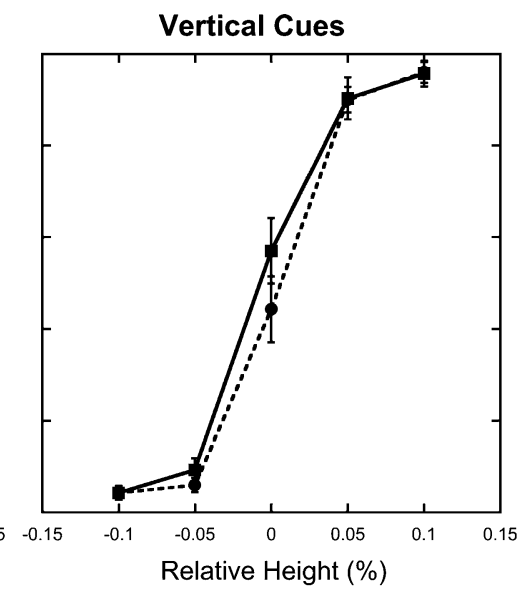

b

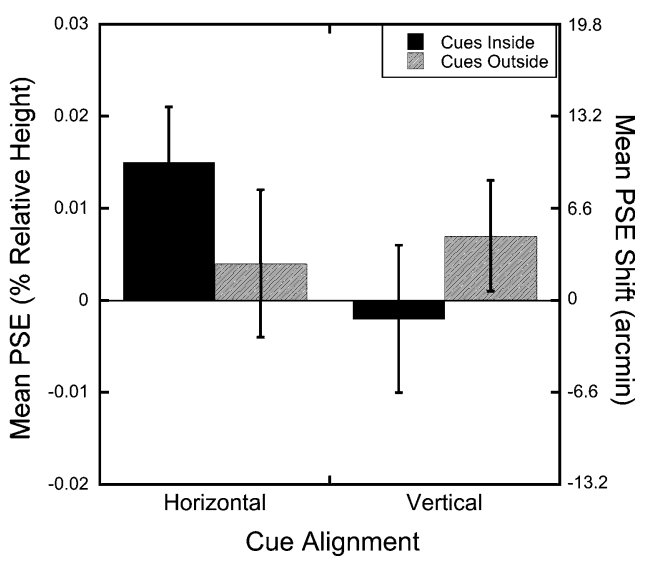

Fig. 3 Results from Experiment 1. (a) Mean percentages of "taller" responses for cues presented along the horizontal and vertical meridians as a function of the relative height of the ovals and the cue position, for the ovals with a horizontal radius of $11^{\circ}$. Trials in which the cues were presented inside the oval contour are shown as squares and solid lines, and trials in which the cues were presented outside the oval contour are shown as circles and dotted lines. (b) Mean estimated points of subjective equality (PSE) as a function of cue alignment and cue position. The right $y$-axis shows PSE shift in arcminutes of a degree. Error bars represent $\pm 1 S E$ 
aligned cues. Here, placing the cues inside the oval contour resulted in a smaller PSE relative to when they were placed outside the contour, suggesting that vertically aligned cues inside the contour increased the perceived height of the oval. Thus, the global structure of an object appears to be stretched or squeezed along the dimension parallel to the attentional cues. Experiment 2 further supported this conclusion.

\section{Experiment 2}

It is possible that the results of Experiment 1 were due to a complex response bias or to some interaction between the contours of the cues and oval that was not due to attentional repulsion. To rule these out, Experiment 2 presented the ovals either simultaneously with the cues (simultaneous condition) or before the cues (postcue condition). If the results are due to a response bias, the same crossover found in Experiment 1 should be replicated in both timing conditions. If the results are due to an interaction between the contours of the oval and the cues present in the display, the same crossover should be observed in at least the simultaneous condition. Conversely, if the distortions in perceived shape depend on the cues attracting attention, the effect should require that the cues precede the oval in time, and the results of Experiment 1 should not be replicated here.

\section{Method}

Participants A new set of 26 undergraduates, selected as before, participated in this experiment. There were 16 females, and the mean age was $20.73 \pm 3.94$ years.
Materials and procedure The stimuli and task were the same as in Experiment 1. Fourteen of the participants completed the postcue condition. In this condition, each trial started with the fixation cross for $500 \mathrm{~ms}$, followed by a 500-ms blank screen. The oval was then presented for $100 \mathrm{~ms}$, followed by a blank screen for $100 \mathrm{~ms}$, and the cues for $50 \mathrm{~ms}$. The other 12 participants completed the simultaneous-cue condition. On every trial here, the fixation cross was shown for $500 \mathrm{~ms}$, followed by a blank screen for $500 \mathrm{~ms}$, and then both the cues and the oval together for $100 \mathrm{~ms}$.

\section{Results and discussion}

The percentage of "taller" responses was calculated for each participant and condition. As can be seen in Figs. 4a and $5 \mathrm{a}$, for both timing conditions, increasing the physical height of the stimulus led to increases in the percentage of "taller" responses ( $p \mathrm{~s}<.001$ for both). However, in contrast to Experiment 1, the same crossover was not found across the different cue placements. For both timing conditions, the three-way interactions were not significant ( $F_{\mathbf{s}}<1$ for both).

As in Experiment 1, the psychometric functions were fit with cumulative Gaussian functions in order to estimate the PSE. The data were well fit by cumulative Gaussian distributions (average $R^{2}=.98$, range $=.88-1.0$ ). The mean PSEs are shown in Figs. $4 \mathrm{~b}$ and $5 \mathrm{~b}$. As can be seen in Fig. $4 \mathrm{~b}$, for the postcue condition there was no main effect of cue position, $F(1,13)=0.33, p=.58, \eta^{2}=.03$. Cue alignment did influence perceived shape, with ovals appearing taller when the cues were vertically aligned, $F(1,13)=15.48, p=.002, \eta^{2}=.54$. More importantly, the interaction between the two factors did not reach significance, $F(1,13)=3.49, p=.09, \eta^{2}=.21$. Although a a

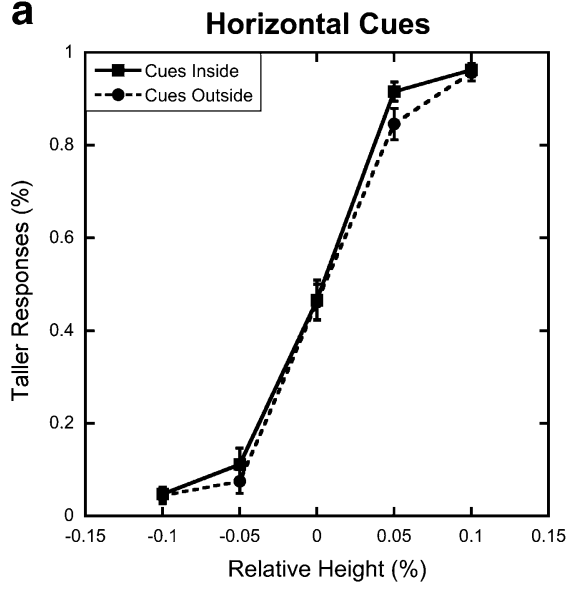

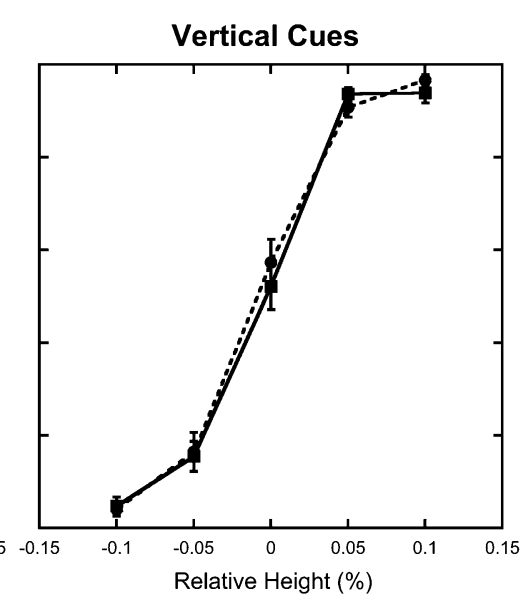

b

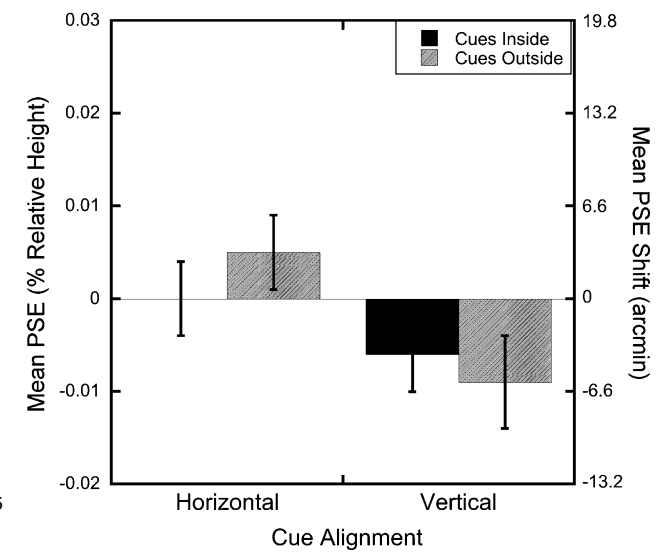

Fig. 4 Experiment 2: Postcue condition. (a) Mean percentages of "taller" responses for cues presented along the horizontal and vertical meridians as a function of the relative height of the ovals and cue position, for the ovals with a horizontal radius of $11^{\circ}$. The same formatting is used here as in Fig. 3. (b) Mean estimated points of subjective equality (PSE) as a function of cue alignment and cue position. The right $y$-axis shows PSE shift in arcminutes of a degree. Error bars represent $\pm 1 S E$. 
possible trend was observed, inspection of Fig. $4 \mathrm{~b}$ shows that the effect was in fact in the direction opposite to that seen in Experiment 1. The main effect of cue alignment is indicative of a response bias in which participants used the alignment of the cues to guide responses for the circle condition. More importantly, the lack of a significant interaction and the fact that the pattern of the means was in the opposite direction, suggesting a possible attraction effect, demonstrates that response bias cannot explain the results of Experiment 1.

The same analyses were performed on the means in the simultaneous condition (Fig. 5b). There was a main effect of cue position, $F(1,11)=5.19, p=.04, \eta^{2}=.32$, with ovals seen as relatively taller when the cues were placed inside the oval contour. The PSEs also showed an elongation of the ovals along the dimension of the cues, $F(1,11)=19.46, p=.001, \eta^{2}=.64$. More importantly, there was no interaction between the two factors, $F<1$. These findings demonstrate that contextual interactions between the cue and the oval cannot explain the repulsion of the oval contour found in Experiment 1.

Might the lack of a replication in Experiment 2 be explained by the recruitment of different participants? To address this question, a 2 (cue alignment) $\mathrm{x} 2$ (cue position) $\mathrm{x} 3$ (timing condition) mixed-design ANOVA was run on the mean PSE estimates, with timing as a between-subjects factor. There was no overall difference in the means across the three timing conditions $(F<1)$. There was a Timing $\mathrm{x}$ Cue Alignment interaction, $F(2,35)=6.85, p=.003$, $\eta^{2}=.28$, driven by the large response bias found in the simultaneous-cue condition that was not observed in Experiment 1, but no Timing x Cue Position interaction, $F(2,35)=1.91, p=.16, \eta^{2}=.10$. Importantly, there was a three-way interaction, $F(2,35)=5.12, p=.01, \eta^{2}=.23$, showing that the relative changes in responses when the cues were placed inside and outside the oval contour across the two cue alignment conditions did vary significantly, depending on the presentation timing of the ovals and cues. This result demonstrates that differences in response variability on the part of participants cannot explain why the interactions of interest in Experiment 1 were not observed in Experiment 2, since such an increase would have eliminated this three-way interaction, given the lack of a significant difference in the overall means across the three timing conditions.

A final concern is whether the results of Experiment 1 reflect a figural aftereffect rather than a distortion due to shifts of attention. Whereas Experiment 1 showed repulsion of the oval contours from the cued locations, Experiment 2 showed something similar to an assimilation effect with simultaneous presentation: The ovals were elongated in the dimension consistent with the cues. Although this could be interpreted as a response bias on the part of the participants, research on Delboeuf concentric circles has shown contrast effects when an inducing circle is shown prior to the target circle, and assimilation effects when it is shown simultaneously (Sagara \& Oyama, 1957). However, this effect cannot explain the present results. First, no interaction was found with the simultaneous presentation, and an assimilation account would still predict differential effects on the shape of the ovals, depending on whether the cues were placed inside or outside the oval contour. Second, previous research (Cooper \& Weintraub, 1970) has shown consistent contrast effects with simultaneous presentation when four nonconcentric circles or quarter-circle arcs are used as inducers. These stimuli are more consistent with the dots used in the present study. Yet no interaction across the two cue positions was found with the simultaneous presentations. a
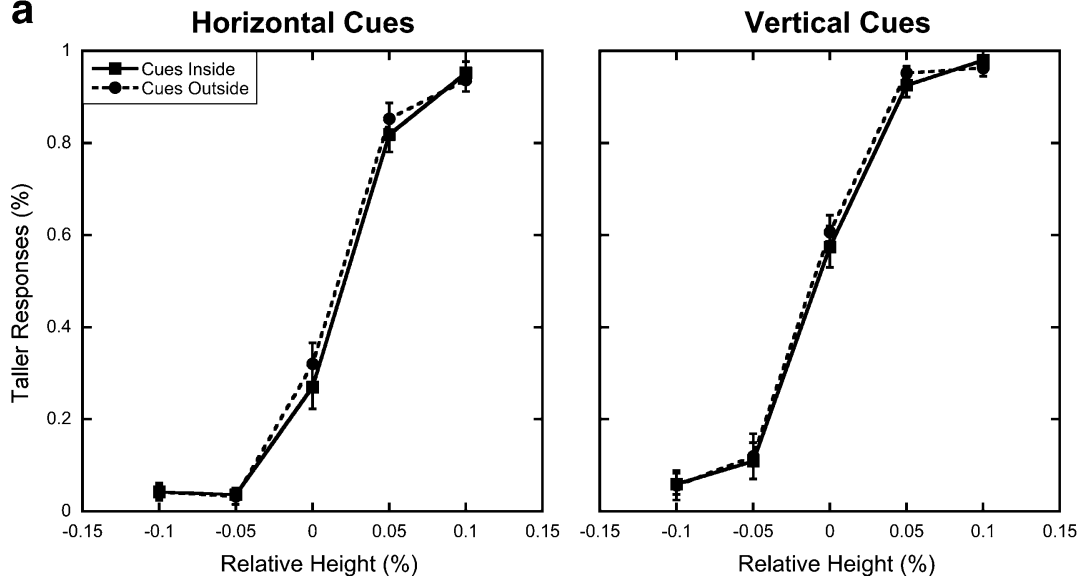

b

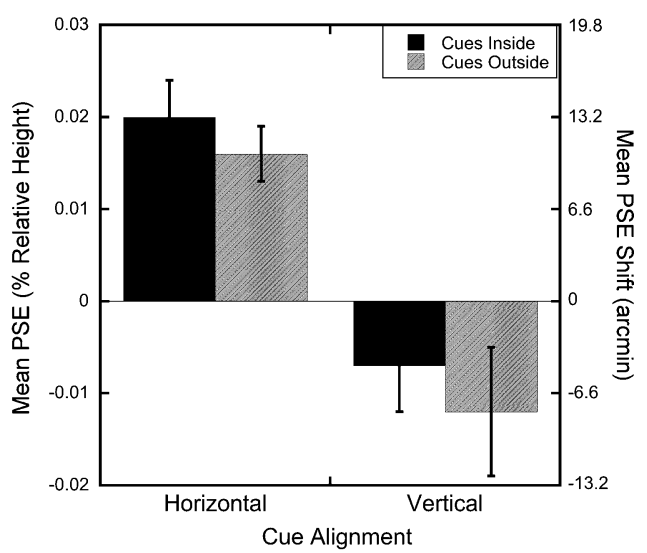

Fig. 5 Experiment 2: Simultaneous condition. (a) Mean percentages of "taller" responses for cues presented along the horizontal and vertical meridians as a function of the relative height of the ovals and cue position, for the ovals with a horizontal radius of $11^{\circ}$. The same formatting is used here as in Fig. 3. (b) Mean estimated points of subjective equality (PSE) as a function of cue alignment and cue position. The right $y$-axis shows PSE shift in arcminutes of a degree. Error bars represent $\pm 1 S E$ 
Another effect, the shape-contrast effect (Suzuki \& Cavanagh, 1998), can be ruled out on the basis of the present results. In the shape-contrast effect, a line presented prior to an oval distorts the perceived shape of the oval such that it is seen as elongated in the direction perpendicular to the line. If we assume that the two cue dots could represent the endpoints of a line, our results may at first appear similar to this. However, Suzuki and Cavanagh (1998) found that ovals were always repelled in the perpendicular direction, regardless of whether the line was longer or shorter than the diameter of the circle. Since these conditions would correspond to the outside and inside cue conditions, the shape-contrast effect cannot explain why the ovals in Experiment 1 appeared elongated along the dimension of the cues when the cues were placed inside the oval contour.

\section{Experiment 3}

Since there are different types of figural aftereffects, a final experiment was conducted in which we put the influence of adapting cues in conflict with the brief cues previously used. In this experiment, we presented two pairs of cues on each trial at the same eccentricity, one set along each meridian. Since the magnitude of aftereffects increases with the duration of the adapting stimulus, we presented one pair of cues for a long duration (1.4 s) and then both pairs for $50 \mathrm{~ms}$ (see Fig. 6a). If the results of Experiment 1 are due to a figural aftereffect, the results should show a repulsion effect away from the locations of the long, adapting cues. On the other hand, if the distortion is due to rapid shifts of attention to the brief cues, the same pattern of errors should be observed.
Method

Participants Eleven undergraduates and one author (F.C.F.), selected as before, participated in this experiment. There were 11 females, and the mean age was $21.1 \pm 3.73$ years.

Materials and procedure The task was the same as Experiment 1, with the following exceptions (see Fig. 6a). Only the perfect circles with $5^{\circ}, 11^{\circ}$, and $17^{\circ}$ radii were presented. The same cue locations were used. The trial structure was changed so that after presentation of the fixation cross, a pair of adapting cues was presented for $1.4 \mathrm{~s}$. Both the adapting cues and the corresponding pair of cues along the opposite meridian were then presented for $50 \mathrm{~ms}$. After a $100-\mathrm{ms}$ blank screen, the target oval was presented for $100 \mathrm{~ms}$. Both the horizontally and vertically aligned cues were presented at the $8^{\circ}$ or the $14^{\circ}$ cue locations, so the cue positions (inside/outside) were the same. The circles were paired with the cues as before, such that the $8^{\circ}$ cues were followed by the $5^{\circ}$ or $11^{\circ}$ circles, and the $14^{\circ}$ cues were followed by the $11^{\circ}$ or $17^{\circ}$ circles. This created eight conditions. Each condition was repeated 25 times.

\section{Results and discussion}

As before, we considered the results for the circle with an $11^{\circ}$ radius. Figure $6 \mathrm{~b}$ shows the mean percentages of "taller" responses when the brief onset cues were aligned along the horizontal or the vertical meridian. As can be seen in Fig. 6b, the introduction of the long, adapting cues along the opposite meridian did not eliminate the repulsion effect observed in Experiment 1. The results from a 2 (cue
Fig. 6 Trial sequence and results from Experiment 3. (a) An example trial sequence showing the timing of the long, adapting cues (vertical) and the brief cues (horizontal) presented before the target circle. The positions of the cues relative to the circle contour (inside vs. outside) were the same on every trial. The cues were always presented on opposite meridians. (b) Mean percentages of "taller" responses for the $11^{\circ}$-radius circle contours as a function of cue alignment and cue position. Cue alignment is defined according to the position of the brief $(50-\mathrm{ms})$ cues. Error bars represent $\pm 1 S E$ a

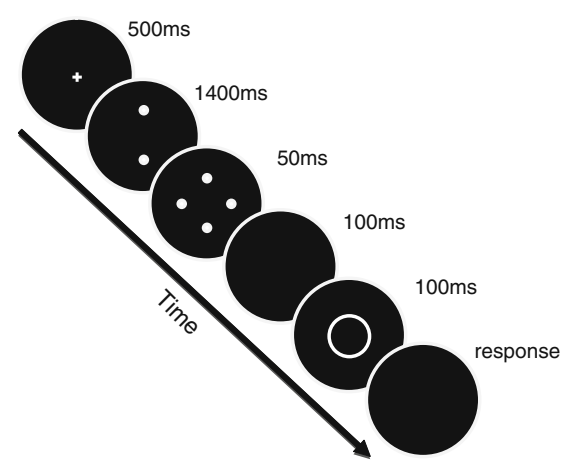

b

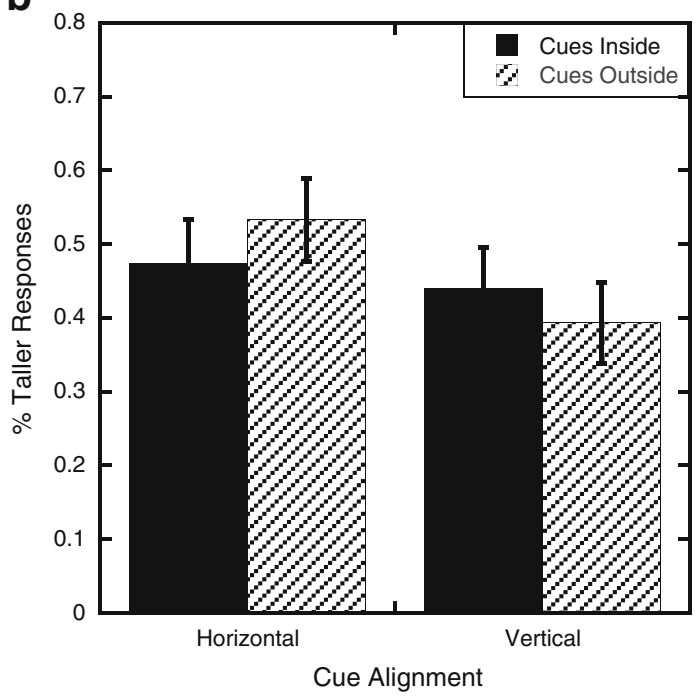


alignment) x 2 (cue position) repeated measures ANOVA show a significant Cue Alignment $x$ Cue Position interaction in the same direction found in Experiment 1, $F(1,11)=4.82, p=.05, \eta^{2}=.31$. There was no main effect of cue position or of cue alignment ( $p s \geq .31)$. Had the repulsion of the oval contours in Experiment 1 been due to rapid adaptation to the cues in the absence of any attention effect, the interaction should have been eliminated, if not reversed, in this experiment. The fact that the responses show a repulsion away from the locations of the brief cues, and thus toward the long adapting cues, supports the hypothesis that the capture of attention by brief cues can distort the perceived shape of subsequently presented targets.

As can be seen in Fig. 6b, the repulsion of the contours away from the brief cues was attenuated relative to Experiment 1. This finding suggests that the long, adapting cues did influence the perception of the circles by reducing the repulsion from the brief cues. Although it is beyond the scope of the present paper to untangle the individual contributions of the long and the brief cues, it should be noted that adaptation and attention effects are not mutually exclusive phenomena; attentional modulations of figural aftereffects have been documented (Yeh, Chen, De Valois, \& De Valois, 1996). However, the present results suggest that rapid shifts of attention dominate perception in the present paradigm.

\section{General discussion}

The present findings demonstrate that not only the location of objects can be distorted by rapid changes in spatial attention, but also the shape of the objects themselves. In Experiment 1, we found that the placement of noninformative cues followed by an oval contour alters the oval's perceived shape. The second experiment demonstrated that this effect depends on the temporal asynchrony between the cues and the oval. In order for part of the oval contour to be repelled away from the cued location, the cues must precede the oval. The third experiment demonstrated that this effect cannot be solely explained by adaptation to the cued location. Collectively, these findings support an account based on changes in attentional orienting induced by the brief cues and their position relative to the target.

How could such involuntary cues lead to a repulsion of the oval contours? Suzuki and Cavanagh (1997) proposed that the orienting of attention toward cue onsets leads to changes in spatial coding by the receptive fields around the cued locations. Surround suppression, receptive field recruitment, or receptive field shrinking were suggested as possible mechanisms that could lead to shifts in the coded location of vernier lines away from the cues. These are all viable possibilities, since changes in neuronal spatial response profiles have been documented following manipulations of focal attention (Connor, Preddie, Gallant \& Van Essen, 1997; Moran \& Desimone, 1985).

As noted by Pratt and Turk-Browne (2003), these mechanism would most likely be restricted to changes in neural processing in early visual cortex, where retinotopy is most evident. Changes in the initial position coding within a retinotopic map can be thought of as altering the structure of the underlying space in which objects exist, since the coding of position across a space provides the information that is used to determine both size and scale (i.e., it defines the metric of a space). Localized errors in position coding will therefore cause distortions in a spatial metric. As a result, such errors would be expected to alter not only the perceived relative locations of multiple objects presented simultaneously, but also the overall structure of a single, two-dimensional object in neighboring regions of that space.

Although the physiological mechanisms underlying the attentional repulsion effect are still open to debate, the present results demonstrate that the effect of rapid changes in spatial attention is not limited to errors in perceived location. Changes in the distribution of spatial attention can also impact the perceived shapes of objects.

Author Note This research was supported by the Veterans Administration, by National Institutes of Health Grant EY016975-05 (L.C.R.), and by an NSF-GRF grant (F.C.F.). L.C.R. has a Senior Research Career Scientist award from the Veterans Administration and is affiliated with the VA Clinical Sciences Research Service, Department of Veterans Affairs Medical Center, Martinez, CA. The authors thank Betty Wang and Hilary Lapping for help with data collection.

Open Access This article is distributed under the terms of the Creative Commons Attribution Noncommercial License which permits any noncommercial use, distribution, and reproduction in any medium, provided the original author(s) and source are credited.

\section{References}

Adam, J.J., Davelaar, E.J., van der Gouw, A., \& Willems, P. (2008). Evidence for attentional processing in spatial localization. Psychological Research, 72, 433-442. doi:10.1007/s00426-007-0126-2

Brainard, D.H. (1997). The Psychophysics Toolbox. Spatial Vision, 10, 433-436. doi:10.1163/156856897X00357

Connor, C. E., Preddie, D. C., Gallant, J. L., \& Van Essen, D. C. (1997). Spatial attention effects in macaque area V4. Journal of Neuroscience, 17, 3201-3214.

Cooper, L.A., \& Weintraub, D.J. (1970). Delboeuf-type circle illusions: Interactions among luminance, temporal characteristics, and inducing-figure variations. Journal of Experimental Psychology, 85, 75-82. doi:10.1037/h0029503 
Jonides, J. (1980). Towards a model of the mind's eye's movement. Canadian Journal of Psychology, 34, 103-112. doi:10.1037/ h0081031

Moran, J., \& Desimone, R. (1985). Selective attention gates visual processing in the extrastriate cortex. Science, 229, 782-784. doi:10.1126/science.4023713

Posner, M.I., Snyder, C.R., \& Davidson, B.J. (1980). Attention and the detection of signals. Journal of Experimental Psychology: General, 109, 160-174. doi:10.1037/0096-3445.109.2.160

Pratt, J., \& Turk-Browne, N.B. (2003). The attentional repulsion effect in perception and action. Experimental Brain Research, 152, 376-382. doi:10.1007/s00221-003-1557-7

Sagara, M., \& Oyama, T. (1957). Experimental studies of figural aftereffects in Japan. Psychological Bulletin, 54, 327-338. doi: $10.1037 / \mathrm{h} 0048995$
Suzuki, S., \& Cavanagh, P. (1997). Focused attention distorts visual space: An attentional repulsion effect. Journal of Experimental Psychology: Human Perception and Performance, 23, 443-463. doi:10.1037/0096-1523.23.2.443

Suzuki, S., \& Cavanagh, P. (1998). A shape-contrast effect for briefly presented stimuli. Journal of Experimental Psychology: Human Perception and Performance, 24, 1315-1341. doi:10.1037/00961523.24.5.1315

Tsal, Y., \& Bareket, T. (1999). Effects of attention on localization of stimuli in the visual field. Psychonomic Bulletin \& Review, 6, 292-296.

Yeh, S.-L., Chen, I.-P., De Valois, K.K., \& De Valois, R.L. (1996). Figural aftereffects and spatial attention. Journal of Experimental Psychology: Human Perception and Performance, 22, 446-460. doi:10.1037/0096-1523.22.2.446 